\title{
The FOXP1, FOXP2 and FOXP4 transcription factors are required for islet alpha cell proliferation and function in mice
}

\author{
Jason M. Spaeth ${ }^{1} \cdot$ Chad S. Hunter $^{1,2} \cdot$ Lauren Bonatakis $^{1} \cdot$ Min Guo $^{1}$ • \\ Catherine A. French $^{3}$ • Ian Slack ${ }^{4}$. Manami Hara ${ }^{5}$ - Simon E. Fisher ${ }^{6,7}$. \\ Jorge Ferrer $^{8} \cdot$ Edward E. Morrisey $^{4} \cdot$ Ben Z. Stanger $^{4} \cdot$ Roland Stein $^{1}$
}

Received: 4 February 2015 / Accepted: 28 April 2015 /Published online: 29 May 2015

(C) Springer-Verlag Berlin Heidelberg 2015

\begin{abstract}
Aims/hypothesis Several forkhead box (FOX) transcription factor family members have important roles in controlling pancreatic cell fates and maintaining beta cell mass and function, including FOXA1, FOXA2 and FOXM1. In this study we have examined the importance of FOXP1, FOXP2 and FOXP4 of the FOXP subfamily in islet cell development and function.

Methods Mice harbouring floxed alleles for Foxp1, Foxp2 and Foxp 4 were crossed with pan-endocrine Pax6-Cre transgenic mice to generate single and compound Foxp mutant mice. Mice were monitored for changes in glucose tolerance by IPGTT, serum insulin and glucagon levels by radioimmunoassay, and endocrine cell development and proliferation by immunohistochemistry. Gene expression and glucosestimulated hormone secretion experiments were performed with isolated islets.
\end{abstract}

Jason M. Spaeth and Chad S. Hunter contributed equally to this study

Electronic supplementary material The online version of this article (doi:10.1007/s00125-015-3635-3) contains peer-reviewed but unedited supplementary material, which is available to authorised users.

Roland Stein

roland.stein@vanderbilt.edu

1 Department of Molecular Physiology and Biophysics, Vanderbilt University Medical School, 723 Light Hall, 2215 Garland Avenue, Nashville, TN 37232, USA

2 Department of Medicine, Division of Endocrinology Diabetes \& Metabolism, University of Alabama at Birmingham, Birmingham, AL, USA

3 Champalimaud Neuroscience Programme, Champalimaud Centre for the Unknown, Lisbon, Portugal
Results Only the triple-compound Foxp 1/2/4 conditional knockout (cKO) mutant had an overt islet phenotype, manifested physiologically by hypoglycaemia and hypoglucagonaemia. This resulted from the reduction in glucagon-secreting alpha cell mass and function. The proliferation of alpha cells was profoundly reduced in Foxp1/2/4 $\mathrm{cKO}$ islets through the effects on mediators of replication (i.e. decreased $C c n a 2, C c n b 1$ and $C c n d 2$ activators, and increased Cdkn1a inhibitor). Adult islet Foxp1/2/4 cKO beta cells secrete insulin normally while the remaining alpha cells have impaired glucagon secretion.

Conclusions/interpretation Collectively, these findings reveal an important role for the FOXP1, 2, and 4 proteins in governing postnatal alpha cell expansion and function.

Keywords Alpha cell · Function · Hypoglycaemia · Proliferation

4 Department of Medicine and Cell and Developmental Biology, University of Pennsylvania, Philadelphia, PA, USA

5 Department of Medicine, University of Chicago, Chicago, IL, USA

6 Language and Genetics Department, Max Planck Institute for Psycholinguistics, Nijmegen, The Netherlands

7 Donders Institute for Brain, Cognition and Behaviour, Radboud University, Nijmegen, The Netherlands

8 Department of Medicine, Imperial Centre for Translational and Experimental Medicine, Imperial College London, London, UK 


$\begin{array}{ll}\text { Abbreviations } \\ \text { 4W } & \text { 4-week-old } \\ \text { 8W } & \text { 8-week-old } \\ \text { ARX } & \text { Aristaless related homeobox } \\ \text { CDK } & \text { Cyclin-dependent kinase } \\ \text { cKO } & \text { Conditional knockout } \\ \text { FOX } & \text { Forkhead box } \\ \text { MAFA } & \text { v-maf Musculoaponeurotic fibrosarcoma oncogene } \\ & \text { family, protein A [avian] } \\ \text { MAFB } & \text { v-maf Musculoaponeurotic fibrosarcoma oncogene } \\ & \text { family, protein B [avian] } \\ \text { PDX1 } & \begin{array}{l}\text { Pancreatic and duodenal homeobox 1 } \\ \text { STZ }\end{array} \\ \text { TF } & \text { Trreptozotocin } \\ & \text { Transcription factor }\end{array}$

\section{Introduction}

The mammalian pancreas is partitioned into two functional compartments: the exocrine pancreas, comprising acinar and ductal cells; and the endocrine pancreas, which has an essential role in maintaining blood glucose homeostasis via secretion of glucose regulatory hormones into the circulation. The endocrine pancreas is comprised of micro-organs, known as the islets of Langerhans, containing five distinct cell populations: beta, alpha, delta, epsilon and pancreatic polypeptide cells that secrete the hormones insulin, glucagon, somatostatin, ghrelin and pancreatic polypeptide, respectively. Type 1 diabetes arises from insulin deficiency resulting from autoimmune-mediated destruction of beta cells, whereas type 2 diabetes is characterised by insulin resistance in peripheral tissues, insulin deficiency and loss of beta cell identity [1]. Although lesser studied, increasing evidence supports the importance of alpha cell dysregulation in the aetiology of both type 1 and type 2 diabetes [2]. Glucagon counteracts insulin action in peripheral tissues to increase hepatic glucose production, thus raising blood glucose levels. Consequently, alpha cell dysfunction confounds glycaemic control in both type 1 and type 2 diabetic patients.

Current efforts are aimed at elucidating mechanisms of endocrine cell lineage determination and expansion as a means of improving protocols for beta cell replacement therapies. In this regard, islet-enriched transcription factors (TFs) have been demonstrated to play an important role in reprogramming terminally differentiated cells and embryonic stem cells (ESCs) into beta-like cells (e.g. v-maf musculoaponeurotic fibrosarcoma oncogene family, protein A [avian] [MAFA], pancreatic and duodenal homeobox 1 [PDX1] and neurogenin 3 [NGN3]) [3-5]. Furthermore, the observation that functional beta cells can arise from differentiated alpha cells has fuelled significant interest in the mechanisms controlling alpha cell development and growth [6]. Interestingly, human alpha cells retain bivalent epigenetic marks on mature beta cell determination genes, MAFA and $P D X 1$, suggesting that they are poised for conversion [7]. Success in generating therapeutic beta cells and understanding normal alpha cell development and function will rely on increased knowledge of the fundamental transcriptional processes underlying pancreatic cell specification and islet cell activity.

Many different TF classes have been found to be critical for pancreatic cell development and function [8]. For example, members of the forkhead box (FOX) superfamily of TFs play a critical role in these processes. FOXA2 is an essential regulator that specifies early pancreatic cell fates, activates a master regulator of pancreas development, $P d x 1$, and is required for euglycaemia in neonatal mice $[9,10]$. Furthermore, the related FOXM1 controls expression of cell cycle factors and augments beta cell mass under conditions of metabolic stress, including pregnancy and partial pancreatectomy $[11,12]$. The function of the FOXP subfamily of regulators is not described in the mammalian pancreas, but FOXP1, FOXP2, FOXP4 were previously reported to be expressed in Xenopus laevis pancreas and eye fields during development [13]. These proteins have overlapping and important roles during mouse central nervous system, lung and heart development [14-16]. In contrast, closely related FOXP3 is critical for $\mathrm{CD} 4^{+}$regulatory $\mathrm{T}$ cell production in mouse and humans [17], but is not expressed in the mammalian pancreas (Human Protein Atlas, www.proteinatlas.org, accessed 15 January 2015, [18]).

To investigate the impact of FOXP1, FOXP2 and FOXP4 on pancreatic endocrine cell formation and function, we generated conditional knockout (cKO) variants using panendocrine Pax6-Cre transgenic mice. Interestingly, only the triple-compound Foxp1/2/4 mutant (Foxp1/2/4 cKO) manifested changes in glucose homeostasis. These mutant mice were born in expected ratios, but developed postnatal hypoglycaemia and hypoglucagonaemia. Foxp $1 / 2 / 4 \mathrm{cKO}$ mice had profoundly reduced numbers of alpha cells $(\sim 85 \%$ reduction) and decreased alpha, delta and beta cell proliferation, with no observed increase in apoptosis. The proliferative defect is mediated through effects on the expression of cell cycle activators (Ccna2, Ccnb1 and Ccnd2) and the cyclin-dependent kinase (CDK) inhibitor, Cdknla. Furthermore, only glucosestimulated glucagon secretion, and not insulin secretion, was defective in adult Foxp1/2/4 cKO islets. We conclude that FOXP1, FOXP2 and FOXP4 are essential for islet alpha cell proliferation and function. This work may impact developing strategies to expand alpha cells for transdifferentiation into therapeutic beta cells as well as understanding the aetiology of alpha cell dysfunction in type 1 and type 2 diabetes.

\section{Methods}

Animals The $\operatorname{Foxp}^{\mathrm{f} / \mathrm{f}}[19,20], \operatorname{Foxp}^{\mathrm{f} / \mathrm{f}}[21], \operatorname{Foxp}^{\mathrm{f} / \mathrm{f}}[19$, 20] and Pax6-Cre [22] mice were maintained on a mixed 
(sv129 and C57BL/6) genetic background. Notably, the Pax6Cre transgene is active in developing eye and islet cells, and does not contain the human growth hormone sequences augmenting islet beta cell mass and insulin content (termed LeCre in Ashery-Padan et al [22]; personal communication: Ashery-Padan, Tel-Aviv University Tel Aviv, Israel). Foxp 1/ $2 / 4$ cKO (Pax6-Cre, Foxp1 $1^{\mathrm{f} / \mathrm{f}}$, Foxp $^{\mathrm{f} / \mathrm{f}}$, Foxp $\left.4^{\mathrm{f} / \mathrm{f}}\right)$ and control littermate mice $\left(F_{\operatorname{oxp}} 1^{\mathrm{f} / \mathrm{f}}, F_{\operatorname{oxp}} 2^{\mathrm{f} / \mathrm{f}}\right.$ and $\left.F_{\operatorname{oxp}} 4^{\mathrm{f} / \mathrm{f}}\right)$ were generated by mating Pax6-Cre with $F_{\operatorname{Oxp}} 1^{\mathrm{f} / \mathrm{f}}, \operatorname{Foxp}^{\mathrm{f} / \mathrm{f}}$ and Foxp $4^{\mathrm{f} / \mathrm{f}}$ mice. The morning of vaginal plug discovery was considered embryonic day (e)0.5. Besides the islet phenotype described herein, various FOXP TF mutants had defects in eye development (electronic supplementary material [ESM] Table 1 and data not shown). The Vanderbilt University Institutional Animal Use and Care Committee approved all animal experiments. Mice were housed in a conventional facility with a $12 \mathrm{~h}$ night/day cycle and had free access to food/water.

Immunohistochemical analyses Staged embryonic and postnatal littermate control and Foxp 1/2/4 cKO pancreatic tissues were fixed in $4 \%$ (vol./vol.) paraformaldehyde, paraffin embedded, and cut to $6 \mu \mathrm{m}$. The Gift of Hope organ procurement organisation (Itsaca, IL, USA) generously provided the de-identified normal and type 2 diabetic cadaver pancreases, and their use was approved by the institutional review board (normal: reference number H94, 59 years old, BMI $25.4 \mathrm{~kg} / \mathrm{m}^{2}$; type 2 diabetes: reference number $\mathrm{H} 78$, 59 years old, BMI $21.2 \mathrm{~kg} / \mathrm{m}^{2}, 14$ year duration of diabetes; reference number H58, 51 years old, BMI $34 \mathrm{~kg} / \mathrm{m}^{2}, 15$ year duration of diabetes). Sections were blocked with $5 \%$ (vol./ vol.) normal donkey serum in $1 \%$ (wt/vol.) BSA/PBS and incubated with primary antibodies overnight at $4{ }^{\circ} \mathrm{C}$. Cyanine dye (Cy)2-, Cy3-, or Cy5-conjugated secondary antibodies (Jackson ImmunoResearch Laboratories, West Grove, PA, 1:2,000) were used for fluorescent detection. Peroxidase staining was performed using the DAB substrate kit (Vector Labs, Burlingame, CA) and counterstained with eosin. Images were collected on a Zeiss Axioimager M2 (Jena, Germany) or an Aperio ScanScope (Leica, Buffalo Grove, IL, USA) whole slide scanner. The following primary antibodies were used: insulin-guinea pig (Dako, Carpinteria, CA, USA; A0564012, 1:1,000); glucagon-mouse (Sigma, St Louis, MO, USA; G2654, 1:2,000); somatostatin-goat (Santa Cruz, Dallas, TX, USA; sc-7819, 1:1,000); Ki67-mouse (BD Pharmingen, San Jose, CA, USA; 550609, 1:1,000); v-maf musculoaponeurotic fibrosarcoma oncogene family, protein B [avian] (MAFB) (Bethyl, Montgomery, TX, USA; IHC-00351,1:1,000); PDX1-goat (kindly provided by C. Wright, Vanderbilt University, 1:20,000); FOXP1 $(1: 1,000)$ and FOXP4 $(1: 1,000)$ antibodies (E. Morrisey).

Hormone cell quantification Six sections ( $240 \mu \mathrm{m}$ apart) from 4-week-old (4W) control and Foxp1/2/4 cKO $(n=3)$ mice were analysed for hormone ${ }^{+}$mass using DAB substrate and counterstained with eosin. The percentage of alpha, beta and delta cell area (relative to whole tissue area) was multiplied by the pancreatic mass to obtain hormone mass. For Ki $67^{+}$proliferative cell counting, the total number of hormone ${ }^{+}$and $\mathrm{Ki} 67^{+}$co-positive cells were counted and divided by the total number of hormone ${ }^{+}$cells.

Islet isolation and RNA preparation Postnatal day (p) 10 Foxp 1/2/4 cKO and control littermates $(n=4)$ were used for pancreatic islet isolations. Islet RNA was isolated using TRIzol (Invitrogen) and treated with DNAse1. RNA quality was analysed by the Vanderbilt Technology for Advanced Genomics Core Facility using an Agilent 2100 Bioanalyzer. The cDNA made using the iScript cDNA synthesis kit (BioRad, 170-8890, Hercules, CA, USA) was subjected to quantitative (q)PCR analysis in a LightCycler 480 II Real-Time PCR System. Gene expression changes between Foxp1/2/4 cKO and control islet RNA was normalised to Gapdh mRNA levels and fold changes calculated using the $\Delta \Delta \mathrm{C}_{\mathrm{t}}$ method. Primers sequences are available upon request.

Islet glucagon and insulin secretion assays Islets from $4 \mathrm{~W}$ Foxp 1/2/4 cKO and control mice were incubated overnight in islet culture media at $37^{\circ} \mathrm{C}$ (RPMI1640, 10\% [vol./vol.] FBS, penicillin/streptomycin, $11 \mathrm{mmol} / 1 \mathrm{D}$-glucose). The next day, islets were incubated for $1 \mathrm{~h}$ at $37^{\circ} \mathrm{C}$ in a baseline solution $\left(1.25 \mathrm{mmol} / 1 \mathrm{CaCl}_{2}, 0.6 \mathrm{mmol} / 1 \mathrm{MgSO}_{4}: 7 \mathrm{H}_{2} \mathrm{O}\right.$, $0.6 \mathrm{mmol} / 1 \mathrm{KH}_{2} \mathrm{PO}_{4}, 2.4 \mathrm{mmol} / \mathrm{l} \mathrm{KCl}, 64.0 \mathrm{mmol} / 1 \mathrm{NaCl}$, $20 \mathrm{mmol} / 1 \mathrm{HEPES} \mathrm{pH} 7.9,5 \mathrm{mmol} / 1 \mathrm{NaHCO}_{3}, 2.8 \mathrm{mmol} / \mathrm{l}$ D-glucose). The secretion of insulin (25 islets per reaction for control and Foxp 1/2/4 cKO) and glucagon (25 control islets and 150 Foxp 1/2/4 cKO islets) was measured after a $45 \mathrm{~min}$ incubation at $37^{\circ} \mathrm{C}$ in stimulation media (baseline: $2.8 \mathrm{mmol} / 1$ or $16.7 \mathrm{mmol} / 1$ glucose for insulin secretion or $1.0 \mathrm{mmol} / 1$ glucose, $10 \mathrm{mmol} / 1 \mathrm{~L}$-arginine for glucagon). Secretion supernatant fraction was collected and hormone content measured from islets lysed in $1.5 \% \mathrm{HCl}, 70 \%$ ethanol (vol./vol.). An RIA was used to determine insulin content in the Vanderbilt Hormone Assay and Analytical Services Core, and an ELISA (RayBiotech, Norcross, GA, USA; EIA-GLU) for glucagon. Data are presented as the percentage of secreted hormone over total hormone content (\% islet content).

IPGTT and plasma serum collection The $4 \mathrm{~W}$ mice $(n \geqq 8)$ were fasted for $6 \mathrm{~h}$ and blood glucose levels were determined using a FreeStyle glucometer. A preparation of $2 \mathrm{mg}$ dextrose/g body weight in sterile PBS was injected intraperitoneally. Blood glucose levels were measured at 15, 30, 60 and 120 min post-injection. Serum was isolated and subjected to glucagon and insulin analysis following a $16 \mathrm{~h}$ fast. 
Statistical analysis All data are expressed as mean \pm standard error of the mean (SEM) and were tested for statistical significance using the Student's two-tail $t$ test.

\section{Results}

\section{Endocrine-specific removal of FOXP1, FOXP2 and FOXP4} results in hypoglycaemia and hypoglucagonaemia FOX proteins have important roles in tissue-specific gene programming in several developing organs including the brain, liver, heart, lung, gut and pancreas. It was previously reported that FOXP1, FOXP2 and FOXP4 are expressed in the mucosal and epithelial layers of the developing intestine by e12.5 in mice $[13,23]$. We investigated the spatio-temporal pancreatic expression of FOXP1 and FOXP4 proteins by immunofluorescence. Both FOXP1 and FOXP4 are broadly expressed throughout the $\mathrm{PDX} 1^{+}$pancreatic endoderm at e12.5 and e15.5 (ESM Fig. 1a, b). Furthermore, FOXP1 and FOXP4 are ubiquitously expressed throughout the endocrine compartment by 3 weeks of age (ESM Fig. 1c) and are present in beta, alpha and delta cells (ESM Fig. 2a-d). FOXP3 was not investigated in this study as there is no evidence for expression in the mammalian pancreas (Human Protein Atlas, www. proteinatlas.org accessed 15 January 2015, [18]), while satisfactory FOXP2 staining could not be obtained using commercially available antibodies.

To evaluate the contributions of Foxp1, Foxp 2 and Foxp 4 to endocrine pancreas development and function, we generated single and compound conditional knockouts using panendocrine-driven Pax6-Cre mice. Recombination of floxed Foxp 1 and Foxp 4 alleles was achieved in hormoneproducing cells by e15.5 as demonstrated by loss of immunoreactive nuclei in Foxp 1/2/4 cKO hormone ${ }^{+}$cells (ESM Fig. 3a, b). Furthermore, Foxp1, Foxp2 and Foxp 4 mRNA levels were significantly reduced in p10 islets (ESM Fig. 3c). As with conditional ablation of other islet TFs (e.g. $P d x 1, N k x 6-1$, Mafa [24-26]), we hypothesised that FOXP1, FOXP2 and/or FOXP4 was involved in maintaining blood glucose levels. Interestingly, only the triple FOXP gene knockout mutant, Foxp1/2/4 cKO, displayed a glucose homeostasis phenotype (Fig. 1), presumably because of functional redundancy of the remaining FOXP factors in the double- and single-gene knockout models. Notably, the pancreas to body weight ratio and overall body weights were unchanged in $4 \mathrm{~W}$ Foxp 1/2/4 cKO mice relative to controls (ESM Fig. 3d-e).

The physiological consequence of removing FOXP1/2/4 on glucose clearance was determined at 4 weeks by IPGTT. Strikingly, mutant mice were glucose tolerant (Fig. 1a, b) though they displayed a significant and non-sex-specific reduction in fasting blood glucose levels relative to controls (Fig. 1c), which persisted in 8 -week-old (8W) adults
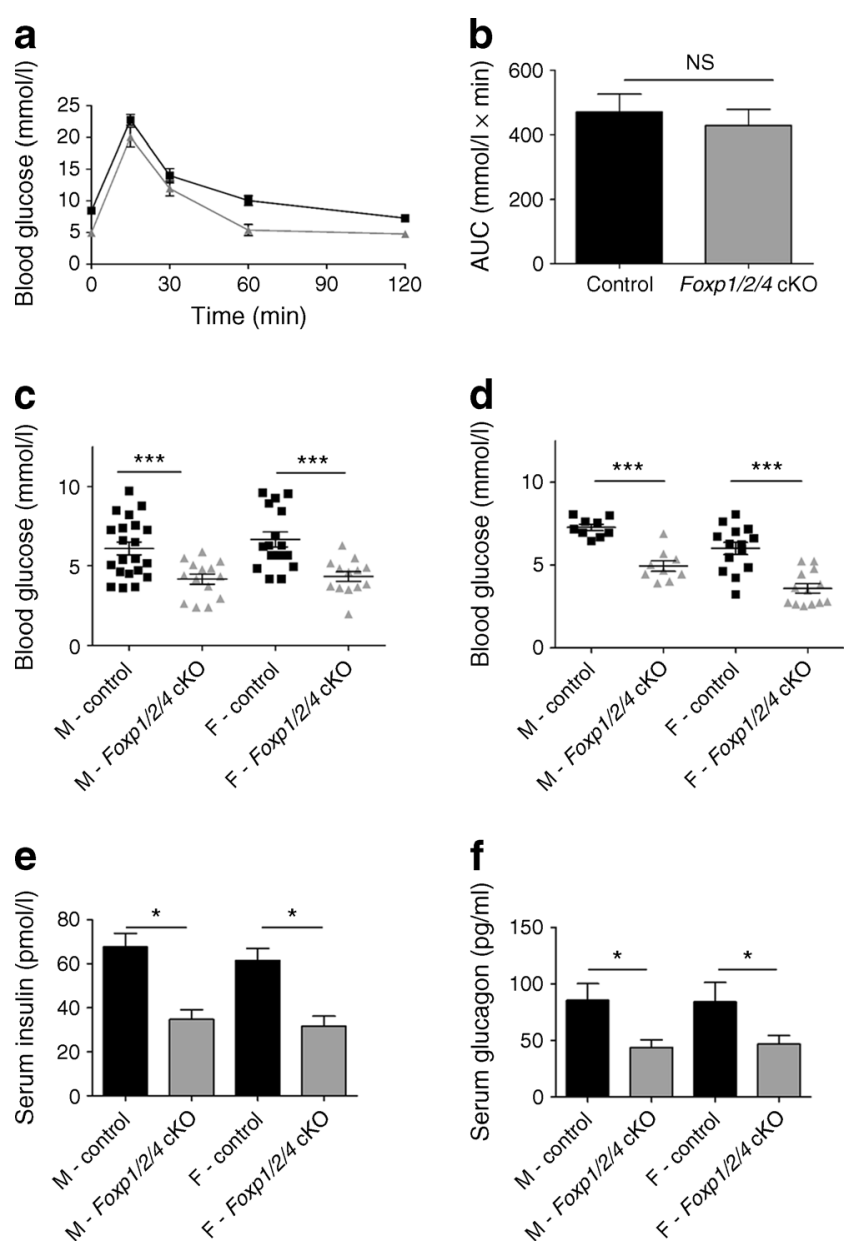

Fig. 1 Foxp 1/2/4 cKO mice are glucose tolerant, but have hypoglycaemia and hypoglucagonaemia. (a) 4W control and Foxp 1/2/4 cKO mice were subjected to IPGTT following $6 \mathrm{~h}$ fasting. Blood glucose measurements were taken at the indicated times. Results are expressed as mean \pm SEM $(n=13$ control [black lines]; $n=8$ Foxp $1 / 2 / 4$ cKO [grey lines]). (b) AUC analysis of IPGTT from (a) (after normalising control and Foxp 1/2/4 cKO time point 0 ) shows no statistical difference in glucose clearance. (c, d) Blood glucose levels from $4 \mathrm{~W}$ (c) and $8 \mathrm{~W}$ (d) control and Foxp 1/2/4 cKO mice after a $6 \mathrm{~h}$ fast $(n=21$, male control; $n=14$, male Foxp $1 / 2 / 4$ cKO; $n=16$, female control; $n=13$, female Foxp $1 /$ $2 / 4 \mathrm{cKO}$ ). (e, f) Serum insulin (e) and glucagon (f) levels from $4 \mathrm{~W}$ control and Foxp 1/2/4 cKO mice following a $16 \mathrm{~h}$ fast $(n=6$, male control; $n=7$, male Foxp $1 / 2 / 4$ cKO; $n=10$, female control; $n=6$, female Foxp $1 / 2 / 4$ cKO). ${ }^{*} p<0.05$ and ${ }^{* * *} p<0.001$ after two-tailed Student's $t$ test. F, female; $\mathrm{M}$, male

(Fig. 1d). We next determined whether the hypoglycaemic phenotype was a consequence of defects in insulin- and/or glucagon-secretion levels. Serum was collected from $4 \mathrm{~W}$ control and Foxp 1/2/4 cKO mice following a $16 \mathrm{~h}$ fast (to exacerbate any potential problems). Serum insulin and glucagon levels were both diminished by nearly $50 \%$ (Fig. 1e, f). The decreased insulin is likely attributed to a normal physiological response to the fasting hypoglycaemia [27], as glucose tolerance in Foxp 1/2/4 cKO mice was normal (Fig. 1a, b). In contrast, the mechanism(s) governing glucagon secretion appeared dysregulated. 
Foxp1/2/4 cKO mice have a dramatic loss of islet alpha cells Immunohistochemical analysis of $4 \mathrm{~W}$ mouse pancreases showed a significant loss of glucagon ${ }^{+}$alpha cells in Foxp 1/2/ $4 \mathrm{cKO}$ pancreases, and no striking differences in beta or delta cells (Fig. 2a-f). Alpha, beta and delta cell mass analysis showed an $\sim 85 \%$ decrease in alpha, an $\sim 50 \%$ decrease in delta and no change in beta cells (Fig. $2 \mathrm{~g}-\mathrm{i}$ ). The average number of insulin $^{+} /$glucagon $^{+} /$somatostatin $^{+}$cells per islet in Foxp 1/2/4 cKO pancreases was significantly decreased, suggesting the islets are smaller in size (ESM Fig. 4a). Furthermore, the reduction of the alpha cell population in 3- and 6-month-old Foxp1/2/4 cKO pancreases shows that this change is permanent (ESM Fig. 4b, c). While it is unclear if serum somatostatin levels are reduced in parallel with delta cell numbers, this would likely not contribute to the hypoglycaemia and hypoglucagonaemia observed, as somatostatin suppresses insulin and glucagon release [28, 29]. Notably, alpha, beta and delta cell numbers were relatively unchanged in e18.5 Foxp 1/ $2 / 4$ cKO pancreases, demonstrating that the change in islet cell production largely occurs postnatally (ESM Fig. $5 \mathrm{a}-\mathrm{c}$ ).

Islet alpha, beta and delta cell proliferation is decreased and cell cycle genes are dysregulated in Foxp1/2/4 cKO islets Proliferative and apoptosis levels in Foxp1/2/4 cKO pancreases were monitored to obtain insight into the mechanism(s) underlying the smaller islet size and severe loss of alpha cells. The level of the Ki67 proliferative marker was negatively affected in p10 Foxp1/2/4 cKO alpha, beta and delta cells (Fig. 3a-e), but not in e18.5 pancreases (ESM Fig. $5 \mathrm{~d}-\mathrm{f}$ ). There was no change in TUNEL apoptosis staining in these samples, and hormone co-expression was not observed at e18.5, p10, 4W or $8 \mathrm{~W}$ (data not shown). Overall, these data suggest that alpha cell deficiency reflects a postnatal proliferative defect, and not cell death or transdifferentiation.

Next, RNA was isolated from hand-picked p10 control and Foxp 1/2/4 cKO islets to examine for changes in cell cycle regulatory genes. Notably, the mRNA levels of Ccna2, Ccnb1 and $C c n d 2$ were significantly reduced, whereas the CDK inhibitor, Cdkn1a, was upregulated (Fig. 3f). FOXP1 was previously shown to reduce Cdk1na expression [30]. Collectively, these observations demonstrate that FOXP1/2/4 regulate the proliferative capacity of pancreatic endocrine cells postnatally.

Glucagon secretion, but not insulin, is abnormal in adult Foxp1/2/4 cKO islets The hypoglycaemic Foxp1/2/4 cKO phenotype was interesting as only $3 \%$ of the mouse islet alpha cell population is apparently required to maintain euglycaemia and normoglucagonaemia [31], yet the Foxp 1/2/ $4 \mathrm{cKO}$ mutant retained roughly $15 \%$ of these cells (Fig. $2 \mathrm{~g}$ ). Islets from $4 \mathrm{~W}$ control (25 islets per reaction) and Foxp1/2/4 cKO (150 islets per reaction, to account for the significant decrease in alpha cell numbers) mice were challenged with $1 \mathrm{mmol} / \mathrm{l}$ glucose plus $10 \mathrm{mmol} / \mathrm{l}$ arginine (a potent glucagon secretagogue [32]) to directly determine if glucagon secretion was defective. These conditions were required because glucagon secretion was not observed in Foxp 1/2/4 cKO islets treated with $1 \mathrm{mmol} / \mathrm{l}$ glucose alone or equivalent number of islets to control (data not shown). The percentage of glucagon secreted from Foxp 1/2/4 cKO islets in the presence of arginine
Fig. 2 Foxp 1/2/4 cKO mice have dramatically reduced islet alpha cell mass. (a-f)

Immunohistochemical analysis of glucagon $(\mathbf{a}, \mathbf{d})$, insulin $(\mathbf{b}, \mathbf{e})$ and somatostatin $(\mathbf{c}, \mathbf{f})$ of $4 \mathrm{~W}$ control and Foxp 1/2/4 cKO pancreases. Scale bars, $40 \mu \mathrm{m}$. (g-i) Alpha (g), beta (h) and delta (i) cell mass was obtained from $4 \mathrm{~W}$ control and Foxp 1/2/4 cKO pancreases ( $n=3$ mice per genotype; six slides per hormone per mouse were analysed). $* p<0.05$ and $* * * p<0.001$ after two-tailed Student's $t$ test
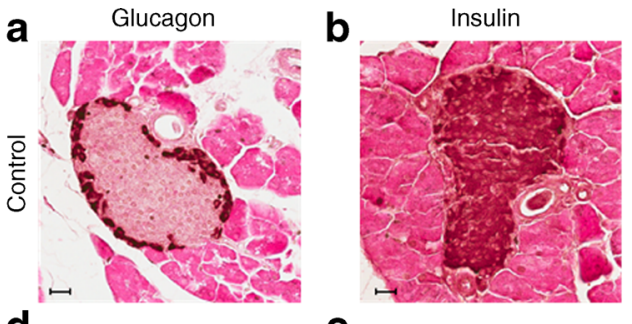

d

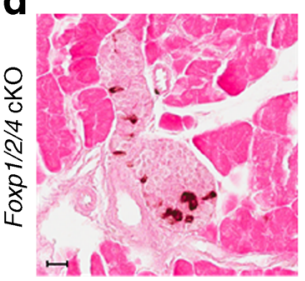

e
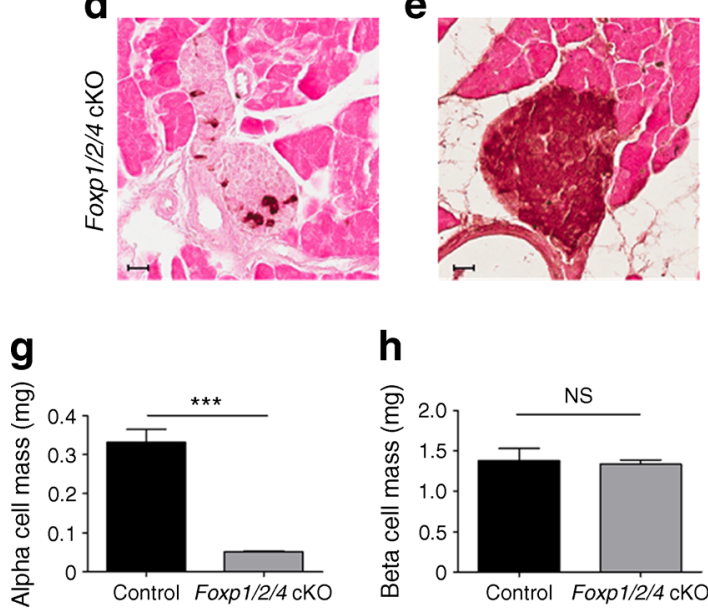

h

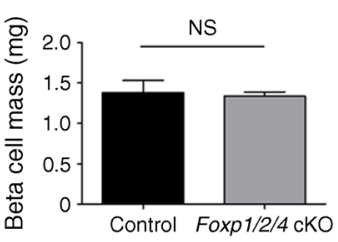

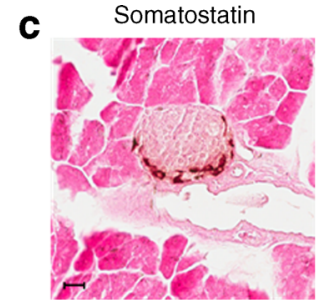

f
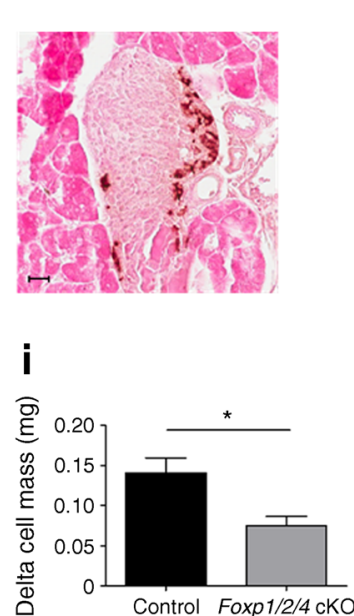
Fig. 3 Islet alpha, beta and delta cell proliferation is decreased and cell cycle gene mRNAs are altered in Foxp 1/2/4 cKO islets. (a, b) p10 control and Foxp 1/2/4 cKO pancreases were stained for insulin, Ki67 and glucagon (a) or insulin, Ki67 and somatostatin (b). In (a): white arrowheads, glucagon ${ }^{+} / \mathrm{Ki} 67^{+}$; yellow arrowheads, Glucagon ${ }^{+} / \mathrm{Ki}^{-} 7^{-}$. In (b): white arrowheads, somatostatin ${ }^{+} / \mathrm{Ki} 7^{+}$; yellow arrowheads, somatostatin ${ }^{+} / \mathrm{Ki}^{-} 7^{-}$. Scale bars, $20 \mu \mathrm{m}$. (c-e) Percentage of proliferating alpha (c), beta (d) and delta cells (e) from control and Foxp 1/2/4 cKO. (f) Quantitative PCR analysis of p10 control and Foxp 1/2/4 cKO islet RNA. mRNA levels in Foxp 1/2/4 cKO islets were normalised against Gapdh and compared directly with normalised control levels arbitrarily set at 1 . Black bars, control; grey bars, Foxp 1/2/4 cKO; $n=4 .{ }^{*} p<0.05 ; * * p<0.01$ and $* * * p<0.001$ after two-tailed Student's $t$ test. GCG, glucagon; Ins, insulin; SST, somatostatin
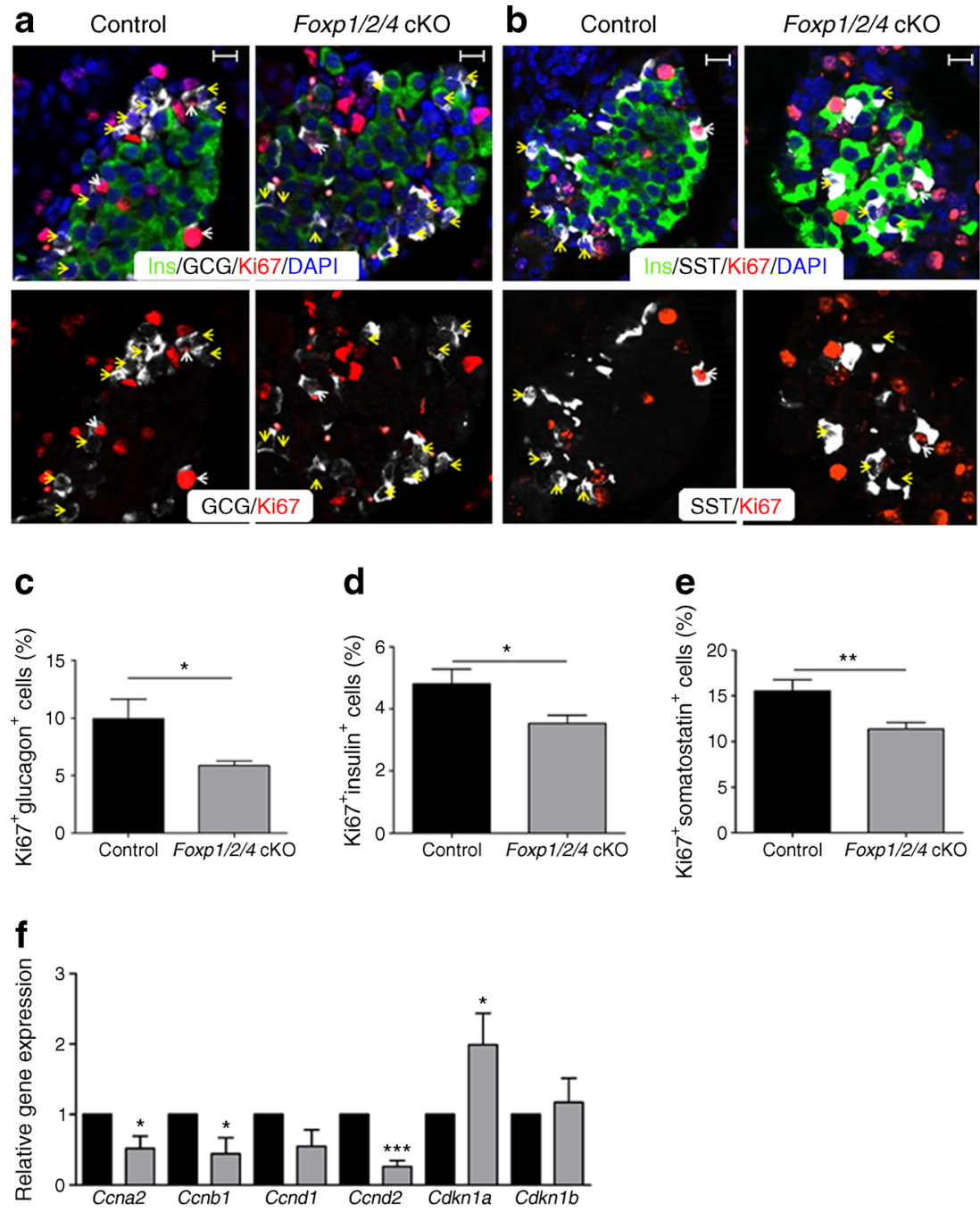

was significantly decreased relative to controls (Fig. 4b). Notably, the reduction of glucagon content per islet (Fig. 4d) was very similar to the reduction in glucagon ${ }^{+}$cell number (Fig. 2g) in Foxp 1/2/4 cKO mice. The alpha cell dysfunction in this mutant could also result from loss of MAFB, a potent driver of glucagon expression and secretion [33], or the likelihood that the alpha cells still express FOXP. However, immunofluorescence analysis demonstrated that glucagon ${ }^{+}$cells are FOXP1/P4 ${ }^{-}$and $\mathrm{MAFB}^{+}$(ESM Fig. 6, Fig. 4a). Importantly, Foxp $1 / 2 / 4 \mathrm{cKO}$ islets exposed to $2.8 \mathrm{mmol} / 1$ (low) and $16.7 \mathrm{mmol} / \mathrm{l}$ (high) glucose secreted similar amounts of insulin (Fig. 4c) and the insulin content per islet was unchanged (Fig. 4e), highlighting the specific nature of the glucagon secretion defects in Foxp 1/2/4 cKO islets.

\section{Discussion}

The glucagon-secreting alpha cell has recently moved to the forefront of efforts to combat type 1 and type 2 diabetes.
Importantly, there are only a few regulators known to specifically impact alpha cell mass or function (aristaless related homeobox [ARX], POU domain, class 3, transcription factor 4 [BRN4], MAFB, FOXA1 and FOXA2 [33-37]); however, the TFs directly impacting alpha cell proliferation have yet to be identified. For example, ARX is critical for maintaining alpha cell identity during development and in neonates, but does not influence alpha cell proliferation [38]. Here, we have shown that conditional removal of FOXP1/2/4 from developing islet endocrine cells significantly impacts glucose homeostasis through reduced postnatal alpha cell proliferation and a dramatic reduction of the alpha cell population.

Fasting hypoglucagonaemia and hypoglycaemia were found in Foxp 1/2/4 cKO mice, which contain $~ 15 \%$ of their alpha cell population. In contrast, Thorel et al concluded that approximately $3 \%$ of normal alpha cells are sufficient to maintain euglycaemia [31]. Comparatively, this suggested that most of the remaining alpha cells in Foxp 1/2/4 cKO islets were dysfunctional and unable to regulate glucagon secretion, which we found to be true (Fig. 4). It is widely accepted that 


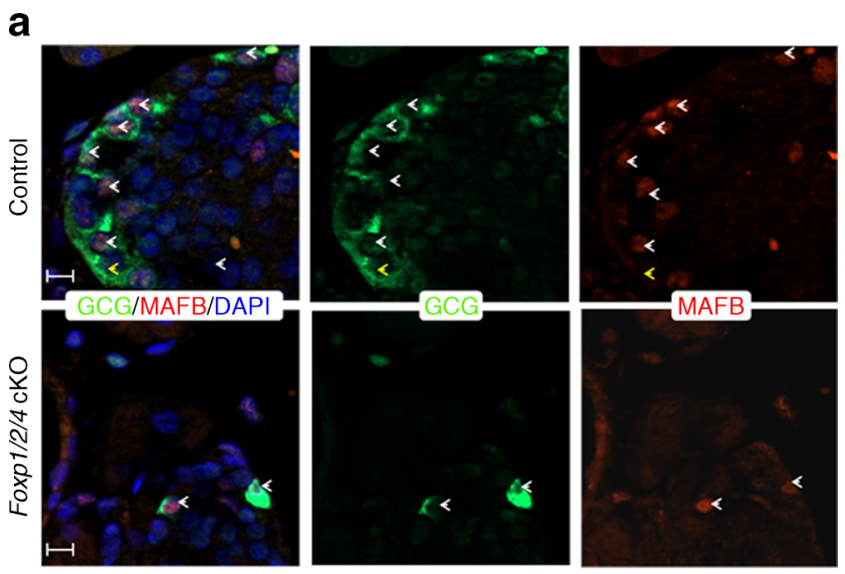

b
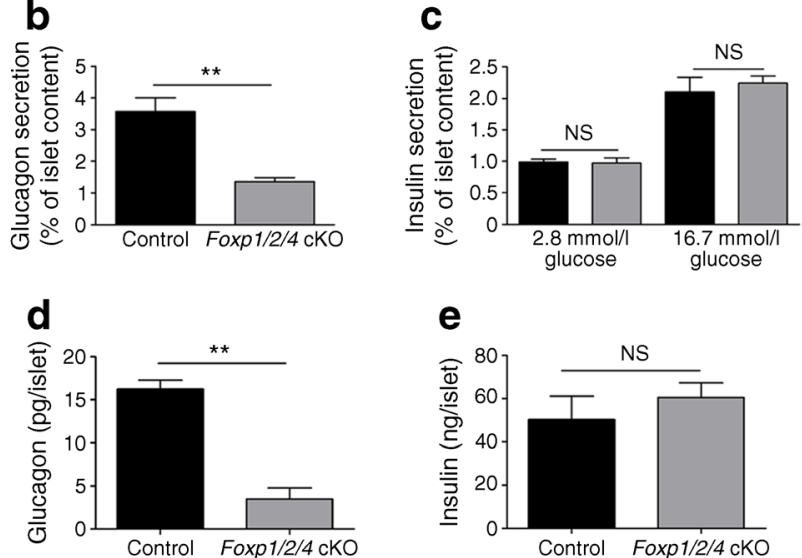

Fig. 4 Foxp 1/2/4 cKO islet alpha cells have defective glucagon secretion. (a) $4 \mathrm{~W}$ control and Foxp 1/2/4 cKO pancreases were monitored by immunofluorescence for glucagon and MAFB. White arrowheads, glucagon $^{+} / \mathrm{MAFB}^{+}$; yellow arrowheads, glucagon ${ }^{+} / \mathrm{MAFB}^{-}$. Scale bars, $20 \mu \mathrm{m}$. (b, c) Islets isolated from $4 \mathrm{~W}$ control (25 islets/reaction) and Foxp1/2/4 cKO (25 islets/reaction, insulin; 150 islets/reaction, glucagon) were incubated with either (b) $1 \mathrm{mmol} / 1$ glucose $+10 \mathrm{mmol} / 1$ arginine (glucagon secretion) or (c) with $2.8 \mathrm{mmol} / 1$ or $16.7 \mathrm{mmol} / 1$ glucose (insulin secretion) for 45 min (black bars, control; grey bars, Foxp 1/2/4 cKO). $n=4$. Data are presented as the percentage of secreted hormone over total hormone content ( $\%$ of islet content). (d, e) Total glucagon (d) and insulin (e) content per islet was calculated in 4W control and Foxp1/ $2 / 4$ cKO islets. ${ }^{*} p<0.01$ after two-tailed Student's $t$ test. GCG, glucagon

hyperglycaemia in diabetes is a consequence of the loss and/or dysfunction of both the islet alpha and beta cell populations $[39,40]$. Without the proper counter-regulatory role of insulin, glucagon action on the liver is unregulated and abnormal hepatic glucose output persists, thus unfavourably exacerbating hyperglycaemia. Mouse models of diabetes induced by streptozotocin (STZ), which is particularly toxic to rodent beta cells, illustrate the action of glucagon in the progression of diabetes. Lee et al have shown that inhibiting total glucagon action impedes the development of STZ-induced diabetes [41]. In contrast, the previously described acute alpha cell ablation model suggests that a few remaining alpha cells mediate normal glucagon signalling and cannot prevent STZ- induced diabetes [31]. As the remaining $15 \%$ of Foxp $1 / 2 / 4$ cKO alpha cells have dysregulated glucagon secretion, it will be interesting to examine if these mutant mice are resistant to STZ-induced diabetes. Importantly, Foxp 1/2/4 cKO hypoglycaemia is presumably not due to the modest decrease in delta cell mass because somatostatin suppresses both insulin and glucagon signalling [28, 29].

Animal models with glucagon action blocked by genetic inhibition of the glucagon receptor $[42,43]$ or reduced active glucagon synthesis [44] have alpha cell hyperplasia. In striking contrast, Foxp1/2/4 cKO mice lack compensatory alpha cell hyperplasia, suggesting that levels of circulating glucagon are either sufficient to prevent alpha cell hyperplasia or, more likely, the remaining alpha cells lack the proliferative capacity. If the latter, it would be expected that alpha cell expansion would not occur upon blocking glucagon signalling in Foxp 1/2/4 cKO mice via treatment with monoclonal antibodies specific for glucagon receptor [45], or by crossing the Foxp $1 / 2 / 4 \mathrm{cKO}$ line with the glucagon receptor knockout mice $\left(\mathrm{Gcgr}^{--}\right)$[42]. Based on earlier findings [30], it is likely that FOXP1, P2, and P4 recruitment of the nuclear receptor co-repressor 2 (NCOR2/SMRT) is required for inhibiting $C d k n 1 a$ promoter activity in islet cells.

The levels of a subset of islet-enriched TFs are reduced in human type 2 diabetic islets, specifically MAFA, MAFB, PDX1 and NK6 homeobox 1 (NKX6.1) [46]. This led us to consider that decreased alpha cell FOXP levels might also contribute to the altered glucagon secretion levels in Foxp 1/ $2 / 4 \mathrm{cKO}$ islets. However, FOXP4 levels appear unchanged in both the alpha and beta cell population (ESM Fig. 7), suggesting that at least FOXP4 levels do not change in type 2 diabetic islets. FOX proteins are particularly important in the developing pancreas and islet function. In this regard, conditional inactivation of Foxa alleles from developing and mature beta cells negatively affects genes involved in metabolic processes, ion transport functions, as well as beta cell enriched enzymes $[10,47]$. Furthermore, FOXA1 and FOXA2 were recently shown to control the expression of genes essential for glucagon production and secretion, including: glucagon, $M a f B$, Brn4 (also known as Pou3f4), Pck2, Nkx2-2, Kir6.1 (also known as Kcnj8), Surl (also known as $A b c c 8$ ), and Gipr [47]. As the FOX family of TFs bind similar DNA response elements, we suspect there is overlap in FOXP1/2/4 and FOXA1/2 gene targets in alpha cells.

Acknowledgements We would like to thank the Vanderbilt Hormone Assay and Analytical Services Core for performing the radioimmunoassays and the Vanderbilt Technology for Advanced Genomics Core Facility for RNA quality analysis. Aperio imaging analysis was performed with assistance from the Vanderbilt Diabetes Research and Training Center (DRTC) and Vanderbilt Cell Imaging Shared Resource.

Funding This work was supported by grants from the National Institutes of Health (5R01DK050203 [RS], R01-HL071589 [EEM], DP2DK083111 [BZS], DK083355 [BZS], 5T32DK07563 [JMS] and DK094842 [CSH]). 
Contribution statement JMS, CSH and RS were responsible for the study design, data acquisition and analysis and wrote the manuscript. LB, $\mathrm{MG}, \mathrm{JF}, \mathrm{MH}$, IS and BZS contributed to data acquisition and analysis. $\mathrm{CAF}, \mathrm{SEF}$ and EEM contributed to the interpretation of data. All authors contributed to the critical revision of the manuscript and approved the final version to be published. RS is the guarantor of this work and, as such, had full access to all the data in the study and takes responsibility for the integrity of the data and the accuracy of the data analysis.

Duality of interest The authors confirm that there is no duality of interest associated with this manuscript.

\section{References}

1. Muoio DM, Newgard CB (2008) Mechanisms of disease: molecular and metabolic mechanisms of insulin resistance and beta-cell failure in type 2 diabetes. Nat Rev Mol Cell Biol 9:193-205

2. Unger RH, Cherrington AD (2012) Glucagonocentric restructuring of diabetes: a pathophysiologic and therapeutic makeover. J Clin Investig 122:4-12

3. Akinci E, Banga A, Tungatt $\mathrm{K}$ et al (2013) Reprogramming of various cell types to a beta-like state by Pdx1, Ngn3 and MafA. PLoS ONE 8:e82424

4. Banga A, Akinci E, Greder LV et al (2012) In vivo reprogramming of Sox 9+ cells in the liver to insulin-secreting ducts. Proc Natl Acad Sci 109:15336-15341

5. Zhou Q, Brown J, Kanarek A et al (2008) In vivo reprogramming of adult pancreatic exocrine cells to $\beta$-cells. Nature 455:627-632

6. Thorel F, Népote V, Avril I et al (2010) Conversion of adult pancreatic alpha-cells to beta-cells after extreme beta-cell loss. Nature 464:1149-1154

7. Bramswig NC, Everett LJ, Schug J et al (2013) Epigenomic plasticity enables human pancreatic alpha to beta cell reprogramming. J Clin Investig 123:1275-1284

8. Pan FC, Wright C (2011) Pancreas organogenesis: from bud to plexus to gland. Dev Dyn 240:530-565

9. Gao N, LeLay J, Vatamaniuk MZ et al (2008) Dynamic regulation of Pdx1 enhancers by Foxa1 and Foxa2 is essential for pancreas development. Genes Dev 22:3435-3448

10. Sund NJ (2001) Tissue-specific deletion of Foxa2 in pancreatic beta cells results in hyperinsulinemic hypoglycemia. Genes Dev 15: 1706-1715

11. Ackermann Misfeldt A, Costa RH, Gannon M (2008) Beta-cell proliferation, but not neogenesis, following $60 \%$ partial pancreatectomy is impaired in the absence of FoxM1. Diabetes 57:3069-3077

12. Zhang H, Zhang J, Pope CF et al (2010) Gestational diabetes mellitus resulting from impaired beta-cell compensation in the absence of foxm1, a novel downstream effector of placental lactogen. Diabetes 59:143-152

13. Schön C, Wochnik A, Rößner A et al (2006) The FoxP subclass in Xenopus laevis development. Dev Genes Evol 216:641-646

14. Rousso DL, Gaber ZB, Wellik D et al (2008) Coordinated actions of the forkhead protein Foxp1 and Hox proteins in the columnar organization of spinal motor neurons. Neuron 59:226-240

15. Li S (2004) Advanced cardiac morphogenesis does not require heart tube fusion. Science 305:1619-1622

16. Shu W, Lu MM, Zhang Y et al (2007) Foxp2 and Foxp1 cooperatively regulate lung and esophagus development. Development 134:1991-2000

17. Schubert LA, Jeffery E, Zhang Y et al (2001) Scurfin (FOXP3) acts as a repressor of transcription and regulates $\mathrm{T}$ cell activation. J Biol Chem 276:37672-37679
18. Pontén F, Jirström K, Uhlen M (2008) The human protein atlas-a tool for pathology. J Pathol 216:387-393

19. Feng X, Ippolito GC, Tian L et al (2010) Foxp1 is an essential transcriptional regulator for the generation of quiescent naive T cells during thymocyte development. Blood 115:510-518

20. Li S, Wang Y, Zhang Y et al (2012) Foxp1/4 control epithelial cell fate during lung development and regeneration through regulation of anterior gradient 2. Development 139:2500-2509

21. French CA, Groszer M, Preece C et al (2007) Generation of mice with a conditional Foxp2 null allele. Genesis 45:440-446

22. Ashery-Padan R, Marquardt T, Zhou X, Gruss P (2000) Pax6 activity in the lens primordium is required for lens formation and for correct placement of a single retina in the eye. Genes Dev 14:27012711

23. Lu MM, Li S, Yang H, Morrisey EE (2002) Foxp4: a novel member of the Foxp subfamily of winged-helix genes co-expressed with Foxp1 and Foxp2 in pulmonary and gut tissues. Mech Dev 119: S197-S202

24. Gannon M, Ables ET, Crawford L et al (2007) Pdx-1 function is specifically required in embryonic beta cells to generate appropriate numbers of endocrine cell types and maintain glucose homeostasis. Dev Biol 314:406-417

25. Schaffer AE, Taylor BL, Benthuysen JR et al (2013) Nkx6.1 controls a gene regulatory network required for establishing and maintaining pancreatic beta cell identity. PLoS Genet 9:e1003274

26. Artner I, Hang Y, Mazur M et al (2010) MafA and MafB regulate genes critical to beta-cells in a unique temporal manner. Diabetes 59:2530-2539

27. Goodner CJ, Conway MJ, Werrbach JH (1969) Control of insulin secretion during fasting hyperglycemia in adult diabetics and in nondiabetic subjects during infusion of glucose. J Clin Invest 48: $1878-1887$

28. Liljenquist JE, Mueller GL, Cherrington AD et al (1977) Evidence for an important role of glucagon in the regulation of hepatic glucose production in normal man. J Clin Invest 59:369-374

29. Cherrington AD, Lacy WW, Chiasson JL (1978) Effect of glucagon on glucose production during insulin deficiency in the dog. J Clin Investig 62:664-677

30. Jepsen K, Gleiberman AS, Shi C et al (2008) Cooperative regulation in development by SMRT and FOXP1. Genes Dev 22:740-745

31. Thorel F, Damond N, Chera S et al (2011) Normal glucagon signaling and alpha-cell function after near-total alpha-cell ablation in adult mice. Diabetes 60:2872-2882

32. Gerich JE, Charles MA, Grodsky GM (1976) Regulation of pancreatic insulin and glucagon secretion. Annu Rev Physiol 38:353388

33. Artner I, Le Lay J, Hang Y et al (2006) MafB: an activator of the glucagon gene expressed in developing islet alpha-and beta-cells. Diabetes 55:297-304

34. Heddad Masson M, Poisson C, Guérardel A, et al. (2014) Foxa1 and Foxa2 regulate alpha-cell differentiation, glucagon biosynthesis, and secretion. Endocrinology 155:3781-3792

35. Kaestner K, Katz J, Liu Y et al (1999) Inactivation of the winged helix transcription factor HNF3alpha affects glucose homeostasis and islet glucagon gene expression in vivo. Genes Dev 13:495-504

36. Lee CS, Sund NJ, Behr R et al (2005) Foxa2 is required for the differentiation of pancreatic alpha-cells. Dev Biol 278:484-495

37. Courtney M, Gjernes E, Druelle N et al (2013) The inactivation of Arx in pancreatic alpha-cells triggers their neogenesis and conversion into functional beta-like cells. PLoS Genet 9:e1003934

38. Wilcox CL, Terry NA, Walp ER et al (2013) Pancreatic alpha-cell specific deletion of mouse Arx leads to alpha-cell identity loss. PLoS ONE 8:e66214

39. Müller WA, Faloona GR, Unger RH (1973) Hyperglucagonemia in diabetic ketoacidosis: its prevalence and significance. Am J Med 54:52-57 
40. Del Prato S, Marchetti P (2004) Beta- and alpha-cell dysfunction in type 2 diabetes. Horm Metab Res 36:775-781

41. Lee Y, Wang M-Y, Du XQ et al (2011) Glucagon receptor knockout prevents insulin-deficient type 1 diabetes in mice. Diabetes 60:391-397

42. Gelling RW, Du XQ, Dichmann DS et al (2003) Lower blood glucose, hyperglucagonemia, and pancreatic alpha cell hyperplasia in glucagon receptor knockout mice. Proc Natl Acad Sci 100: $1438-1443$

43. Longuet C, Robledo AM, Dean ED et al (2013) Liver-specific disruption of the murine glucagon receptor produces alpha-cell hyperplasia: evidence for a circulating alpha-cell growth factor. Diabetes 62:1196-1205
44. Furuta M, Zhou A, Webb G et al (2001) Severe defect in proglucagon processing in islet alpha-cells of prohormone convertase 2 null mice. J Biol Chem 276:27197-27202

45. Gu W, Yan H, Winters KA et al (2009) Long-term inhibition of the glucagon receptor with a monoclonal antibody in mice causes sustained improvement in glycemic control, with reversible alphacell hyperplasia and hyperglucagonemia. J Pharmacol Exp Ther 331:871-881

46. Guo S, Dai C, Guo M et al (2013) Inactivation of specific beta cell transcription factors in type 2 diabetes. J Clin Investig 123:3305-3316

47. Gao N, Le Lay J, Qin W et al (2010) Foxa1 and Foxa2 maintain the metabolic and secretory features of the mature beta-cell. Mol Endocrinol 24:1594-1604 